\title{
A AÇÃO LÚDICA NA INTERNAÇÃO HOSPITALAR INFANTIL SOB A PERSPECTIVA DOS PROFISSIONAIS DA SAÚDE
}

\author{
Giovana Cugini Sanches ${ }^{1}$, ORCID ID 0000-0002-6453-7283; Maria Victória Penteado Vargas ${ }^{1}$, ORCID ID 0000- \\ 0002-6774-6224; João Carlos Diniz², ORCID ID 0000-0001-6438-3336
}

\section{FILIAÇÃO}

(1) Universidade de Taubaté, Medical Student

(2) Universidade de Taubaté, Medical Student

(3) Universidade de Taubaté, Professor do Departamento de Pediatria

\section{AUTOR CORRESPONDENTE}

Giovana Cugini Sanches; giovana.cugini@gmail.com; Rua do Colégio Nossa Senhora do Bom Conselho, № 12, Jardim das Nações - Taubaté, São Paulo; Universidade de Taubaté.

MENSAGENS-CHAVE
$\begin{gathered}\text { A internação hospitalar pediátrica necessita de um atendimento diferente das demais especialidades da área da } \\ \text { saúde, focado na humanização. }\end{gathered}$
Apesar de os profissionais concordarem com a humanização, a maioria não se aprofunda em cursos para
implantar a ludoterapia.
O reconhecimento de um ponto fraco na abordagem pediátrica possibilita o aprimoramento dos profissionais na
atenção à criança.
Não buscar conhecimento adequado sobre a ludoterapia implica em uma falha médica, sendo essencial mudar
esse cenário em prol das crianças.

\section{RESUMO}

INTRODUÇÃO: A experiência da internação e doença pediátrica tem um grande impacto sobre o físico e psicológico da criança. Com isso, a ludicidade deve ser vista como um instrumento de auxílio, pois proporciona humanização do atendimento e melhor aceitação ao tratamento e ambiente hospitalar. Objetiva-se descrever a importância da atividade lúdica sob a ótica dos profissionais da saúde na humanização do atendimento pediátrico e em sua formação acadêmica. METODOLOGIA: Estudo transversal, quantitativo e descritivo, mediante aplicação de questionário estruturado pelos próprios pesquisadores para os profissionais de saúde atuantes na Clínica Pediátrica, seguindo as normas de prevenção da pandemia COVID-19. RESULTADO: Foram entrevistados 31 profissionais de saúde, dos quais $54,8 \%$ conhecem a ludoterapia. Dos participantes, $93 \%$ praticam algum tipo de brincadeira ao abordar o paciente pediátrico e, dentre esses, $80,6 \%$ não tiveram treinamento. Ressalta-se que $77,4 \%$ acreditam que a frequência que a criança vai à biblioteca interfere com a sua percepção do hospital e $96,7 \%$ concordam que a ludoterapia deve ser inserida na formação do profissional. DISCUSSÃO: É inato do profissional ter atitudes lúdicas com a criança mesmo sem o treinamento, o que mostra a naturalidade com a qual o lúdico pode ser inserido na prática pediátrica hospitalar. Ainda assim, ressalta-se a importância de um treinamento específico, visando a minimização de riscos e a humanização do atendimento à criança. CONCLUSÃO: A maioria dos entrevistados utiliza do lúdico e reconhece sua relevância na abordagem integral na pediatria, apesar de não ter preparação técnica sobre o assunto. Além disso, a experiência dos profissionais sugere que a brinquedoterapia ameniza o impacto da internação no desenvolvimento da criança e melhora sua adesão ao tratamento. Assim, demonstra-se a importância da sua discussão teórico-prático em centros universitários e complexos hospitalares, além da ampliação do estudo para avaliar outros parâmetros do impacto da ludoterapia. 


\section{ABSTRACT}

INTRODUCTION: The experience of hospitalization has a major impact on the child's physical and psychological balance. Thus, the use of creative acts should be seen as an instrument to help hospitalized children, as it provides humanization of re and better acceptance of the treatment and hospital environment. The objective is to describe the importance of the playful activity under the perspective of health professionals in the humanization of pediatric care and in academic education. METHODOLOGY: This is a cross-sectional, quantitative and descriptive study that applied a questionnaire structured by the researchers themselves to the health professionals working at the Pediatric Clinic of $X X X$, following the norms of prevention considering the current COVID-19 pandemic. RESULTS: 31 health professionals were interviewed, $54.8 \%$ of whom are familiar with play therapy. Of the participants, $93 \%$ practice creative activity when approaching the pediatric patient and, among these, $80.6 \%$ had no training. Of the professionals, $77,4 \%$ believe that the frequency that the child accesses the toy library interferes with their perception of the hospital environment and $96,7 \%$ agree that play therapy should be inserted in the training of health professionals. DISCUSSION: It is innate for professionals to have playful attitudes with the child even without training, which shows the naturalness with which playfulness can be inserted in hospital practice. Still, the importance of specific training is emphasized, aiming at minimizing risks and humanizing child care. CONCLUSION: Most of the interviewees use the ludic and recognize its relevance in pediatrics, despite not having technical preparation. In addition, the experience of professionals suggests that toy therapy mitigates the impact of hospitalization on the child's development and improves their adherence to treatment. Thus, from the perspective of professionals, the importance of their theoretical-practical discussion in university centers and hospital complexes is demonstrated, among the necessity of expansion of the study to assess other parameters of the impact of play therapy.

\section{KEYWORDS: Playtherapy; Humanization of Assistance; Pediatrics}

\section{INTRODUÇÃO}

O lúdico compreende as atividades que objetivam proporcionar prazer e alegria ao indivíduo1. Essas atividades possibilitam o desenvolvimento integral da criança, desde a parte neuropsicomotora até às competências sociais $(2,3)$, sendo de extrema importância para a saúde física e mental, aprendizado e função cognitiva (4). Assim, a criança torna-se capaz de criar e reinventar o mundo, explorar seus limites, libertar seus pensamentos e expressar seus sentimentos, desenvolvendo habilidades essenciais, como coordenação motora, comunicação, raciocínio, consciência corporal, entre outras $(5,6)$.

A experiência do complexo adoecimento-hospitalização impacta o desenvolvimento físico e afetivo da criança, pois, além de sofrer com a sua condição clínica instável, a rotina hospitalar representa também sua despersonalização. Isso ocorre porque está associada à perda da privacidade, afastamento de entes queridos e do ambiente de conforto, ruptura com o cotidiano e tratamentos invasivos e dolorosos $(2,6)$. Dessa forma, torna-se necessária a inserção da ludoterapia nas práticas hospitalares, a qual integra as atividades lúdicas ao cuidado e tratamento, reconhecendo seu potencial terapêutico em reduzir esses impactos negativos na criança $(7,8)$.

O benefício do riso e do bom-humor na saúde foi demonstrado através da monitorização de experiências cômicas para entender o que acontece no organismo1,8,9. Foi demonstrado o aumento quantitativo em $0,05 \%$ das células de defesa Natural Killer (NK) após 21 indivíduos assistirem a um filme caricato (10). Ademais, foi documentado aumento na concentração salivar da imunoglobulina A $(\lg A)$ de indivíduos após assistirem um vídeo humorístico, em contraste com nenhuma alteração após os mesmos assistirem um vídeo didático e sem cunho satírico (11). Ambas são componentes da resposta imunológica, de modo que as células NK, elemento da imunidade inata, têm grande importância na vigilância imunológica contra agentes infecciosas e neoplásicos, enquanto a $\lg \mathrm{A}$ é o principal anticorpo nas mucosas, neutralizando microrganismos e toxinas (12).

Quanto ao sistema neuroendócrino, o riso diminui os hormônios envolvidos na fisiologia do estresse, como cortisol e epinefrina, por facilitar a oxigenação e causar o relaxamento musculo-esquelético $(13,14)$. Além disso, tem ação analgésica por aumentar a disponibilidade de endorfina, neurotransmissor e hormônio de efeito opióide (5.) Tal fato é corroborado por outro estudo, que revelou que a maioria das crianças $(n=57,5 \%)$ que relataram dor e desconforto anteriores a visita dos palhaços, indicaram uma redução dos sintomas sem o uso de analgésicos e que apenas $8 \%$ revelaram que o humor piora com a intervenção (10).

Outro benefício da inserção das atividades lúdicas no contexto hospitalar é a promoção do desenvolvimento psicológico, social e moral da criança, ajudando-a a encarar suas frustrações e expressar seus sentimentos; além de apresentarse como uma distração para os momentos difíceis $(1,5)$. Nesse contexto, é importante destacar o contar histórias, também chamada de biblioterapia, principalmente utilizando contos de fadas, os quais trazem aproximação com a criança ao tratarem de forma criativa problemas pouco discutidos. Medo, perda, morte e abandono, são alguns dos sentimentos retratados na maioria dos contos infantis, e causam na criança uma identificação com os personagens e sua situação, sendo um facilitador da expressão de emoções. Assim, auxilia os profissionais a trabalharem holisticamente com os pacientes, uma vez que melhora seu desenvolvimento expressivo, cognitivo e social $(13,15)$.

Tendo em vista todos esses aspectos positivos da ludoterapia, foi realizado este estudo após as visitas realizadas pela Companhia da Alegria (CIA). A CIA de XXX foi fundada em 2000 sob administração do Diretório Acadêmico, e, em 2017, tornou-se um Projeto de Extensão da Universidade, com atividades 
realizadas pelos graduandos de Medicina aos sábados. Para seus integrantes, o brincar é um ato indispensável ao desenvolvimento do ser humano, que auxilia na compreensão e enfrentamento das adversidades da hospitalização, proporcionando aprendizagem coletiva e ludicidade ao público infantil internado na Clínica Pediátrica do XXX. Essa, possui uma brinquedoteca, a qual representa o principal local de intervenção da CIA em suas visitas, onde há área de TV, mesa para atividades, alfabeto, tabuada, brinquedos e espaço para brincadeiras individuais e em grupo. Em relação às crianças restritas ao leito, parte dos brinquedos são levados até 0 paciente e são elaboradas outras atividades como música, leitura e teatro. Com isso, apesar de todo o estresse do ambiente hospitalar, o paciente consegue expressar seus sentimentos e revelar aos profissionais alguma necessidade física ou emocional que não era possível apenas com a linguagem.

O lúdico serve como um meio de comunicação e vínculo entre os pilares profissional-família-criança, auxiliando no entendimento da doença, na adesão às medidas terapêuticas e na própria reabilitação, pois, ao resgatar o controle sobre o próprio corpo, a criança encontra forças para lutar $(1,5,14)$. Assim, este estudo tem a proposta de descrever o valor terapêutico das atividades lúdicas na hospitalização infanto-juvenil, a partir de uma visão dos profissionais atuantes na Clínica Pediátrica do XXX, tendo em vista que esta abordagem é pouco discutida, tanto na literatura, quanto durante a formação desses profissionais.

\section{METODOLOGIA}

Trata-se de um estudo transversal, quantitativo e descritivo. A técnica de amostragem foi do tipo não-probabilística e por conveniência, sendo composta por 31 profissionais de saúde (Tabela 1) atuantes na Clínica Pediátrica do Hospital Universitário XXX. Seu tamanho foi escolhido por representar a média de profissionais de saúde que rotacionam no serviço em período diurno. Foi aplicado um questionário estruturado pelos próprios pesquisadores, com variáveis quantitativas do tipo contínua. O tratamento dos dados foi feito por meio de análise descritiva.

O instrumento utilizado foi um questionário com maioria de perguntas fechadas ( $\operatorname{sim} /$ não), sobre o conhecimento e o uso de intervenções lúdicas com crianças sob a ótica dos profissionais que atuam na clínica pediátrica. Esse tema foi abordado inicialmente com questionamentos sobre especialização, conhecimento da atuação da CIA e do termo ludoterapia. Também foi interrogado sobre a prática de brincadeiras e se houve treinamento, além de sua percepção sobre o impacto dessa atividade lúdica sob o quadro geral do paciente e sua adesão ao tratamento. Ademais, foram levantados questionamentos sobre a capacidade do profissional de lidar com o sofrimento, sua visão sobre a importância da família durante a internação, o impacto do ambiente hospitalar no desenvolvimento da criança e se o brinquedo o ameniza. Como tópico final, foi interrogado se o profissional acredita que ludoterapia deveria ser inserida na formação acadêmica da área da saúde.
O questionário foi aplicado na Clínica Pediátrica do $X X X$, onde há 15 leitos disponíveis, entre eles cirúrgicos e clínicos, tendo uma média mensal de 37 pacientes internados da faixa etária desde o nascimento até 11 anos, 11 meses e 29 dias. Nesse local, há normas para prevenção da COVID-19 devido a pandemia vigente, que consistem em precaução de contato e aerossóis como uso de EPI e distanciamento social. Os pesquisadores adotaram as mesmas, bem como uso de álcool em gel antes e depois da entrevista e uso individual de caneta esferográfica. O critério de inclusão foi a atuação no setor da Clínica Pediátrica em algumas das profissões supracitadas pelo tempo mínimo de um mês. O critério de exclusão foi a recusa da autorização mediante termo de consentimento livre e esclarecido.

\begin{tabular}{clc}
\hline Área & Profissionais & $\begin{array}{c}\text { Quantidade de } \\
\text { profissionais } \\
\mathbf{n}(\%)\end{array}$ \\
\hline Medicina & Pediatria & $2(6,4 \%)$ \\
& $\begin{array}{c}\text { Residente de } \\
\text { Pediatria }\end{array}$ & $8(25,9 \%)$ \\
&
\end{tabular}

Residente de $3(9,7 \%)$ Cirurgia

Enfermagem Enfermeiro

Técnico de
Enfermagem

Auxiliar de

Enfermagem

$7(22,6 \%)$

Fisioterapia Fisioterapeuta

$1(3,2 \%)$

\begin{tabular}{ll} 
Nutrição & Nutricionista \\
\hline
\end{tabular}

Total 31

Tabela 1. Número de profissionais entrevistados na Clínica Pediátrica do Hospital Universitário XXX divididos por cursos dentro da área da saúde e suas especializações (Fonte própria).

A coleta de dados compreendeu um período de 2 meses. $O$ estudo observou as diretrizes da Resolução 466/12, do Conselho Nacional de Saúde e foi aprovado pelo Comitê de Ética em Seres Humanos da XXX.

\section{RESULTADOS}

Foram entrevistados 31 profissionais de saúde, dos quais $54,8 \%$ (17) conhecem a ludoterapia e $93,5 \%$ (29) praticam algum tipo de brincadeira ao abordar o paciente pediátrico. A maioria dos integrantes da pesquisa, $80,65 \%$ (25), não tiveram nenhum tipo de treinamento técnico sobre a ludoterapia (Tabela 2). Ao comparar pela área de atuação, os profissionais que tiveram 
algum treinamento de práticas lúdicas foram pediatra, residente de pediatria, enfermeiro e técnico de enfermagem (Tabela 3). Todos os profissionais acham importante a presença de familiares durante a internação. Todos os que praticam atividade lúdica acreditam que a participação da família durante alguma forma de intervenção é importante.

\begin{tabular}{lccc}
\hline Prática de & Treinados & Não Treinados & $\begin{array}{c}\text { Total } \\
\text { atividades }\end{array}$ \\
lúdicas & $\mathbf{n}(\%)$ & $\mathrm{n}(\%)$ & $\mathrm{n}(\%)$
\end{tabular}

Sim

$6(19,35 \%)$

$23(74,20 \%)$

29

$(93,5$

$\%)$

Não

0

$2(6,45 \%)$

2

$(6,5 \%$

) amostra de 31 entrevistados.

Diante dos resultados obtidos, sugere-se que é inato do ser humano e, principalmente, ao profissional da pediatria, ter atitudes lúdicas com a criança mesmo sem ter o treinamento técnico. Essa observação pode ser vista no fato de que 23 $(74,2 \%)$ dos entrevistados praticam algum tipo de brincadeira ao abordar o paciente pediátrico, mesmo sem ter conhecimento específico sobre a ludoterapia (Tabela 2). Esses profissionais se proporem espontaneamente a essa atitude, mostra o instinto acolhedor do ser humano frente à criança hospitalizada, e a naturalidade com a qual as atividades lúdicas podem ser inseridas na abordagem, facilitando a comunicação e o vínculo com o paciente, mas ainda assim ressalta-se a importância de um treinamento específico, visando a minimização de riscos para a criança e a humanização do atendimento $(2,3,6)$.

Apesar de todos os entrevistados concordarem que a ludoterapia auxilia na melhora do quadro clínico geral da criança e sua adesão ao tratamento, é visto que a maioria deles não têm uma preparação teórico-prática. Apenas seis profissionais entrevistados tinham na formação cursos para abordagem recreativa, o que demonstra a discrepância entre a necessidade desse facilitador frente a oferta e interesse dos profissionais pela especialização. Discernindo por área de atuação (Tabela 3), percebe-se que a carência é generalizada nas áreas, considerando que os enfermeiros e os técnicos de enfermagem foram os únicos com um terço dos profissionais pesquisados com treinamento. Destaca-se a falta de treinamento entre pediatras, residentes da pediatria e residentes de cirurgia, sugerindo que as formações focam, em grande parte, na doença e em seu tratamento cirúrgico e medicamentoso, e não no doente de forma holística. Há falta de incentivo das instituições (hospitalares e educativas), bem como de procura por parte dos acadêmicos, de aprendizado de técnicas lúdicas como o uso do brinquedo, música e jogos, contação de histórias, e incentivo à leitura. Essa capacitação precisa estar em concordância com a proposta lúdica de melhora da abordagem, vínculo e aceitação do tratamento $(5,6,10)$.

Considerando que 21 dos profissionais da saúde atuantes no setor pediátrico não acham o ambiente hospitalar adequado para desenvolvimento neuropsicomotor (DNPM), ressalta-se o impacto do complexo adoecimento-hospitalização no crescimento físico e afetivo do paciente, o qual está também em formação de personalidade. Isso ocorre, principalmente, porque a rotina hospitalar pode representar uma ruptura com as relações interpessoais e coletivas das crianças, associado ao sofrimento pela doença e tratamentos invasivos $(2,5,8)$.

Como o Hospital XXX possui em sua enfermaria pediátrica uma brinquedoteca (que representa o principal foco de ação da CIA da Alegria), os profissionais conseguem uma visão comparativa do comportamento da criança antes e após a ida ao espaço recreativo. Entre os profissionais (10) que consideram 0 ambiente hospitalar adequado para 0 crescimento da criança, 6 deles percebem uma melhora adjacente com o uso de brinquedos. Ademais, a maioria dos entrevistados (24) acredita que a frequência de acesso à brinquedoteca é diretamente proporcional a melhora da 
percepção do paciente sobre o ambiente hospitalar, uma vez que o espaço proporciona uma abstração da sua condição enferma e familiarização com ambientes sociais, por conta da interação com outras crianças, participação da família e uso de brinquedos estimulantes da criatividade $(5,6)$.

A brinquedoteca é, portanto, um instrumento recreativo importante, porém não é totalmente inclusiva. Os pacientes em condições especiais como acamados e/ou em isolamento não conseguem usufruir de seus benefícios, sendo papel da equipe de saúde trazer o lúdico ao leito. Logo, a presença da mesma não pode ser o único método de interação e abstração infantil, sendo necessário valorizar o treinamento do profissional de saúde, de forma que ele possa trazer atividades, músicas e expressões criativas para o quarto $(8,9,10)$.

Neste estudo, um terço dos profissionais não se sentiam aptos para lidar com o sofrimento enfrentado pela criança $e$ seus familiares. Visto que, durante a internação podem ocorrer momentos de adversidades como dor, choro, desconforto, medo e ansiedade, tornam-se necessários métodos de distração da criança com técnicas que diminuam o sofrimento, principalmente durante o exame físico e exames invasivos. Isso é corroborado por estudos que demonstram 0 efeito do riso no sistema neuroendócrino, com redução de cortisol e epinefrina, além de ação analgésica por maior disponibilidade de endorfina $(5,13,14)$.

\begin{tabular}{|c|c|c|c|}
\hline Área & $\begin{array}{c}\text { Profissionai } \\
\mathbf{s}\end{array}$ & $\begin{array}{c}\text { Treinados } \\
\text { (n) }\end{array}$ & $\begin{array}{c}\text { Não } \\
\text { Treinados } \\
\text { (n) }\end{array}$ \\
\hline \multirow[t]{3}{*}{ Medicina } & Pediatria & 1 & 1 \\
\hline & $\begin{array}{l}\text { Residente de } \\
\text { Pediatria }\end{array}$ & 1 & 7 \\
\hline & $\begin{array}{l}\text { Residente de } \\
\text { Cirurgia }\end{array}$ & 0 & 3 \\
\hline \multirow{3}{*}{$\begin{array}{l}\text { Enfermage } \\
\mathrm{m}\end{array}$} & Enfermeiro & 2 & 4 \\
\hline & $\begin{array}{l}\text { Técnico de } \\
\text { Enfermagem }\end{array}$ & 2 & 1 \\
\hline & $\begin{array}{l}\text { Auxiliar de } \\
\text { Enfermagem }\end{array}$ & 0 & 7 \\
\hline Fisioterapia & $\begin{array}{l}\text { Fisioterapeut } \\
\text { a }\end{array}$ & 0 & 1 \\
\hline Nutrição & Nutricionista & 0 & 1 \\
\hline Total & & 6 & 25 \\
\hline
\end{tabular}

Tabela 3. Profissionais que receberam ou não treinamento técnico para a prática da ludoterapia no contexto hospitalar da pediatria (Fonte própria).
Salienta-se que esse estudo pode ter sido afetado pela pandemia vigente do COVID-19, uma vez que reduziu o número da amostra. As atividades da Companhia da Alegria e o acesso à brinquedoteca também estão reduzidas pelo distanciamento social previsto pelas recomendações de prevenção. Além disso, a rotatividade de profissionais também pode influenciar na falha de abordagem e treinamento médico e da enfermagem. Essas adversidades foram contornadas ao entrevistar profissionais que compunham o serviço antes da pandemia ou que tiveram contato mesmo com as atividades da CIA reduzidas.

\section{CONCLUSÃO}

Este estudo objetivou descrever a importância da atividade lúdica hospitalar, sob a ótica dos profissionais da saúde atuantes na Clínica Pediátrica XXX, na humanização do atendimento infanto-juvenil e em sua formação.

A partir da perspectiva dos entrevistados, pode-se concluir que a maioria deles utiliza do lúdico e reconhece sua relevância na abordagem integral da criança, apesar da maioria não ter preparação teórico-prática sobre o assunto. Também pode-se observar que os profissionais de saúde, baseado em sua experiência, consideram que a brinquedoterapia ameniza o impacto da internação no desenvolvimento da criança e melhora sua adesão ao tratamento. Visto que a terapia holística na hospitalização infanto-juvenil é pouco abordada na literatura e durante a formação desses profissionais, torna-se relevante a sua prática e discussão em centros universitários.

Dessa forma, considera-se importante a procura de treinamento e participação em palestras relacionados ao tema, além de incentivo a ações como a da CIA da Alegria, adjacentes ao acolhimento da equipe multidisciplinar. É necessária a ampliação do estudo para avaliar outros parâmetros do impacto da ludoterapia a nível de dor, bemestar, tempo de internação e impacto no prognóstico com dosagem de marcadores bioquímicos ou imunológicos, que corroborem tal benefício.

\section{CONFLITOS DE INTERESSE}

Os pesquisadores afirmam que não há conflitos de interesse nesta pesquisa.

\section{FINANCIAMENTO}

O financiamento deste trabalho foi realizado por meios próprios dos autores.

\section{REFERÊNCIAS}

1. 1. Rodrigues $A A$, Abuquerque VB. The playing and caring: the look of occupational therapy on the playful behavior of children in prolonged hospitalization. Revista Interinstitucional Brasileira de Terapia Ocupacional REVISBRATO [internet]. 2020 [cited 2020 Mar 5];4:27-42. Available from: https://revistas.ufri.br/index.php/ribto/article/view/26293. doi:10.47222/2526-3544.rbto26293 
2. Bragio J. The meaning of being an educator of/in the playrooms of the children's hospital in Vitória/ES: a study based on the concepts of experience, narrative and care [dissertation]. Vitoria: Postgraduate Course in Education, Federal University of Espírito Santo; 2014 [cited 2020 Mar 8]. 142p. Available from: http://repositorio.ufes.br/handle/10/2413.

3. Sena AGG. Joy doctors and health professionals: the hospital clown in the caregiver's perception [thesis doctorate]. Belo Horizonte: Nursing Course, Federal University of Minas Gerais; 2011 [cited 2020 Mar 8]. 98p. Available from: http://hdl.handle.net/1843/GCPA8KUHE9.

4. Lima KYN, Santos VEP. The playful as a strategy in the care of children with cancer. Natal: Federal University of Rio Grande do Norte. Rev Gaúcha Enferm [internet]. 2015 [cited 2020 Mar 10]; Jun;36(2):76-81. Available from:

https://seer.ufrgs.br/RevistaGauchadeEnfermagem/artic le/view/51514/34191.

5. Rodrigues CEM. Importance of play in the psychological impact of child hospitalization [dissertation]. Cidade da Praia: Nursing Course, Jean Piaget University of Cape Verde; 2013 [cited 2020 Jun 2]. $74 \mathrm{p}$. Available from: https://core.ac.uk/download/pdf/38682701.pdf.

6. 6. Luz JH. Educate-Play-Care: a problematizing proposal for teaching the toy/therapeutic toy for the undergraduate nursing course [thesis doctorate]. Florianópolis: Nursing Course, Federal University of Santa Catarina; 2015 [cited 2020 Jun 2]. 293p. Available

from:

https://repositorio.ufsc.br/xmlui/handle/123456789/1573 91.

7. Berk LS, Felten DL, Tan SA, Bittman BB, Westengard $J$. Modulation of neuroimmune parameters during the eustress of humor-associated mirthful laughter. Altern Ther Health Med. 2001 [cited 2020 Jun 5];7(2):62-76. Available

from: https://www.ncbi.nlm.nih.gov/pubmed/11253.

8. Abreu GRF. The (good)humor therapy in health care processes. Salvador: Bahian Nursing Magazine. 2011 [cited Jun 25];25(1):69-74. Available from: https://portalseer.ufba.br/index.php/enfermagem/article/ view/5062.

Doi: http://dx.doi.org/10.18471/rbe.v25i1.5062.

9. Sousa LMM, et al. Humor intervention in the nursepatient interaction. Revista Brasileira de Enfermagem [Internet]. 2019 [cited 2020 Jun 29];72(4):1078-1085. Available

from: https://www.scielo.br/j/reben/a/pYDKXdFnnT3kSrMTSr dZCrp/abstract/?lang=en. doi: 10.1590/0034-71672018-0609.

10. Queiroz MYF, Machado RIL, Cordeiro M. Influence of clown therapy on hospitalized child care. Readings Physical Education and Sports [internet], Buenos Aires. 2014 [cited 2020 Jul 15];18(2):1-7. Available from: http://www.repositorio.ufc.br/handle/riufc/10236.
11. Zaguini CGS, Bianchin MA, Junior RVL, Chueire RHMF. Assessment of the playful behavior of children with cerebral palsy and the perception of their caregivers. Acta Fisiatrica [internet]. 2011 [cited 2020 Jul 15];18(4):187-91. Available from: https://www.revistas.usp.br/actafisiatrica/article/downloa $\mathrm{d} / 103663 / 102116$.

12. Alcântara PL, et al. Effect of interaction with clowns on vital signs and non-verbal communication of hospitalized children. Revista Paulista de Pediatria [internet]. 2016 [cited 2020 Aug 20];34(4)432-438. Available from: https://doi.org/10.1016/i.rppede.2016.02.011.

13. Abbas AK; Lichtman AW. Imunologia Básica. 4. ed. Rio de Janeiro: Elsevier; 2013:328.

14. Sridharan K, Sivaramakrishnan G. Therapeutic clowns in pediatrics: a systematic review and meta-analysis of randomized controlled trials [internet]. Eur $\mathrm{J}$ Pediatr. 2016 [cited 2020 Nov 15];175(5):1353-1360. Available from: https://doi.org/10.1007/s00431-016-2764-0.

15. Mota PC. The Influence of Play Therapy on Therapeutics of Hospitalized Children [dissestation] Brasília: Postgraduate of Nursing Course, Catholic University of Brasília. 2008 [cited 2020 Nov 16]. 53p. Available from: https://repositorio.ucb.br/jspui/bitstream/10869/5007/1/P riscilla\%20Caetano\%20Mota.pdf. 